\title{
NF-kB-Induced Loss of MyoD Messenger RNA: Possible Role in Muscle Decay and Cachexia
}

\author{
Denis C. Guttridge, ${ }^{1}$ Marty W. Mayo, ${ }^{1}$ Lee V. Madrid, ${ }^{1,2}$ \\ Cun-Yu Wang, ${ }^{1 *}$ Albert S. Baldwin Jr. ${ }^{1,2,3 \dagger}$
}

MyoD regulates skeletal muscle differentiation (SMD) and is essential for repair of damaged tissue. The transcription factor nuclear factor kappa $B(N F-\kappa B)$ is activated by the cytokine tumor necrosis factor (TNF), a mediator of skeletal muscle wasting in cachexia. Here, the role of NF- $\mathrm{BB}$ in cytokine-induced muscle degeneration was explored. In differentiating C2C12 myocytes, TNF-induced activation of NF-KB inhibited SMD by suppressing MyoD mRNA at the posttranscriptional level. In contrast, in differentiated myotubes, TNF plus interferon- $\gamma(\mathrm{IFN}-\gamma)$ signaling was required for NF-KB-dependent down-regulation of MyoD and dysfunction of skeletal myofibers. MyoD mRNA was also downregulated by TNF and IFN- $\gamma$ expression in mouse muscle in vivo. These data elucidate a possible mechanism that may underlie the skeletal muscle decay in cachexia.

The vertebrate skeletal muscle differentiation (SMD) program is under the strict control of the myogenic bHLH transcription factor family (MyoD, Myf5, myogenin, and MRF4) and of a second class of transcription factors termed myocyte enhancer factor-2 (MEF2A through MEF2D) (1,2). MyoD and Myf5 are expressed in proliferating undifferentiated myoblasts and, upon growth factor withdrawal, are activated to initiate SMD that ultimately leads to the fusion of myoblasts into multinucleated myotubes $(1,2)$. Although mice lacking MyoD develop normally (3), MyoD-deficient skeletal muscle is severely impaired in its ability to regenerate after tissue injury, which suggests a specific role for MyoD in the replenishment of lost muscle (4).

$\mathrm{NF}-\kappa \mathrm{B}$ is a transcription factor expressed in a variety of cell types, including mature muscle (5), and it inhibits SMD by regulating the expression of cyclin D1 (6). The cytokine tumor necrosis factor (TNF) is a potent activator of NF- $\mathrm{B}$, causing it to translocate to the nucleus and bind to promoters and enhancers of genes involved in inflammatory and proliferative responses (7-9). TNF is an important mediator of skeletal muscle degeneration associated with cachexia, a debilitating syndrome characterized by extreme weight loss and

\footnotetext{
${ }^{1}$ Lineberger Comprehensive Cancer Center, ${ }^{2}$ Curriculum in Genetics and Molecular Biology, ${ }^{3}$ Department of Biology, University of North Carolina, Chapel Hill, Mason Farm Road, Campus Box 7295, Chapel Hill, NC, 27599-7295, USA.

*Present address: Laboratory of Molecular Signaling, Department of Biologic and Material Science, University of Michigan, Ann Arbor, MI 48109, USA.

†To whom correspondence should be addressed. Email: jhall@med.unc.edu
}

molecular etiology of cachexia, and hence few targets have been identified for therapy. Here we investigate the potential role of $\mathrm{NF}-\kappa \mathrm{B}$ as a downstream effector of TNFmediated skeletal muscle dysfunction.

TNF was recently shown to inhibit skeletal myogenesis in vitro $(6,14)$. We therefore investigated the potential role of NF- $\mathrm{B}$ in this regulatory process. The addition of TNF to mouse C2C12 myocytes completely blocked their SMD program, as evidenced by the reduced expression of the differentiation markers myogenin and the cyclin-dependent kinase inhibitor $\mathrm{p} 21$, and by the complete absence of the late-stage differentiation marker myosin heavy chain (MHC) (Fig. 1A). TNF also caused a severe reduction in MyoD protein levels (Fig. 1B) but had no effect on the transcription factors Myf5 or MEF2D, which are also expressed but are inactive in undifferentiated myoblasts. Stable heterologous expression of MyoD restored the ability of $\mathrm{C} 2 \mathrm{C} 12$ cells to maintain SMD (Fig. 1C) and form myotubes (15) in the presence of TNF, indicating that TNF inhibits myogenesis by down-regulating MyoD. The rapid loss of MyoD protein was preceded by an equally rapid loss of MyoD mRNA (Fig. 1D). In addition, cells devoid of NF- $\mathrm{B}$ activity [through stable expression of the NF- $\mathrm{B}$ inhibitor Iк $\mathrm{B} \alpha(\mathrm{I} \kappa \mathrm{B} \alpha \mathrm{SR})]$ retained $M y o D$ mRNA in the presence of TNF (Fig. 1D), indicating that
Fig. 1. TNF-induced activation of NF- $\mathrm{B}$ inhibits SMD through suppression of MyoD synthesis. (A) $\mathrm{C} 2 \mathrm{C} 12$ cells were switched from growth medium [GM: Dulbecco's modified Eagle's medium-H (DMEM-H) and $20 \%$ fetal bovine serum] to differentiation medium [DM: DMEM$\mathrm{H}, 2 \%$ horse serum, and insulin $(5 \mu \mathrm{g} / \mathrm{ml})]$ and treated with or without TNF (Promega) at $20 \mathrm{ng} / \mathrm{ml}$ for a 72-hour period (to sustain NF- $\mathrm{B}$ activity, TNF was also added at 6,12 , and 24 hours after the initial treatment). At indicated times, wholecell extracts were prepared and probed for SMD markers myogenin and the cyclin-dependent kinase inhibitor p21 (M-225 and C-19, Santa Cruz Biotechnology), MHC (MY-32, Sigma), or $\alpha$-tubulin (Sigma). (B) TNF regulation is specific to MyoD. Extracts from $\mathrm{C} 2 \mathrm{C} 12$ cells in DM treated with or without TNF were probed for MyoD, Myf5 (M-318 and C-20, respectively, Santa Cruz Biotechnology), and MEF2D (Transduction Laboratories). (C) Overexpression of MyoD restores SMD in the presence of TNF. C2C12 cells were infected with pBabepuro virus containing MyoD cDNA, and selected with puromycin $(1 \mu \mathrm{g})$. Clones were screened for their expression of MyoD and p21 (15). Vector control cells and three independent clones-pBabeMyoD1, -4, and -7-were treated with TNF in DM, and extracts were probed for P21 (after 48 hours) and MHC (after 72 hours). (D) TNF regulation of

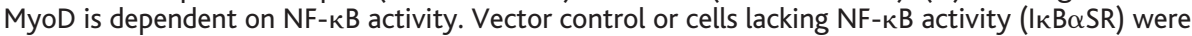
differentiated and treated with TNF. At the indicated times, MyoD and GAPDH mRNA expression was analyzed 
NF- $к \mathrm{~B}$ is a downstream TNF effector that regulates $M y o D$ expression.

To examine which NF- $\mathrm{B}$ subunits were
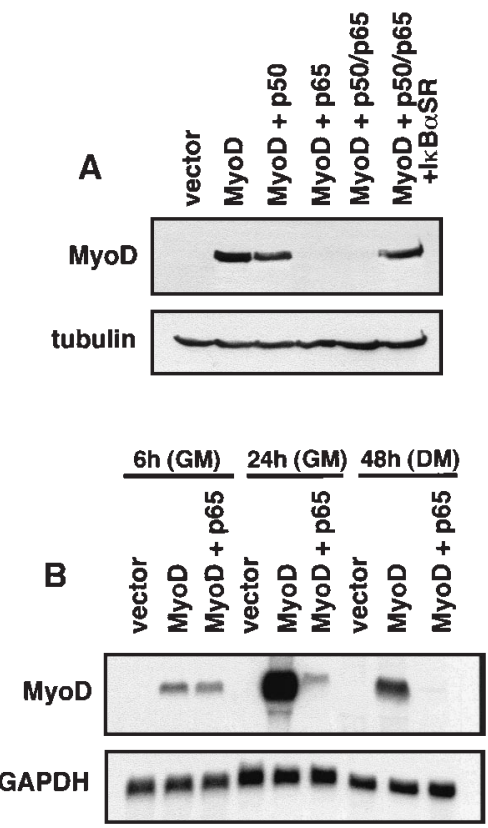

Fig. 2. MyoD is regulated by the $p 65$ subunit of NF-кB. (A) p65 suppresses MyoD expression. 10T1/2 fibroblasts were transfected with MyoD, NF- $\kappa \mathrm{B}$, and $\mathrm{I} \kappa \mathrm{B} \alpha \mathrm{SR}$ expression plasmids (23). Whole-cell lysates were prepared and probed for MyoD and $\alpha$-tubulin. (B) p65 suppresses MyoD mRNA expression. Transfections were repeated in 100-mm culture dishes with $0.5 \mu \mathrm{g}$ of vector or of MyoD expression plasmid, with or without $1.0 \mu \mathrm{g}$ of p65 expression plasmid. Cells were either kept in GM or differentiated in DM, and at indicated times after transfection, RNA was prepared and probed for $M y O D$ and GAPDH mRNA expression. involved in the regulation of $M y o D$, reporter assays were performed in mouse $10 \mathrm{~T} 1 / 2$ fibroblasts. Coexpression of p50 and p65 subunits, or of the p65 subunit alone, strongly blocked MyoD activity, and this effect was reversed with equivalent expression of the I $\mathrm{B} \alpha \mathrm{SR}$ plasmid (16). Examination of whole-cell lysates from these transfections demonstrated that p65 alone was sufficient to dramatically reduce MyoD protein levels (Fig. 2A), and this inhibition was again lost after the expression of $\mathrm{I} \kappa \mathrm{B} \alpha \mathrm{SR}$. In addition, similar to what was observed with TNF treatment of $\mathrm{C} 2 \mathrm{C} 12$ cells, overexpression of p65 reduced $M y o D$ mRNA levels (Fig. 2B). These results demonstrate that TNF regulation of MyoD in $\mathrm{C} 2 \mathrm{C} 12$ cells is dependent on $\mathrm{NF}-\kappa \mathrm{B}$ activity and that the $\mathrm{p} 65$ subunit is sufficient to induce the down-regulation of MyoD mRNA.

These transfection results showed that $\mathrm{p} 65$ regulated $M y o D$ transcripts produced from a heterologous promoter. This suggested that NF- $\kappa \mathrm{B}$ was ultimately targeting the $M y o D$ mRNA itself for down-regulation. We therefore attempted to map the $M y o D$ sequences that rendered the mRNA susceptible to p65mediated down-regulation. Deletion analysis revealed that nucleotides 539 through 914 in the $M y o D$ mRNA were required for this response (Fig. 3A). Introduction of this MyoD fragment into the coding region of the $G A P D H$ gene resulted in a p65-dependent reduction in GAPDH mRNA levels (Fig. 3B), confirming that p65 targets this $M y o D$ sequence. We next examined whether NF-кB inhibition of $M y o D$ mRNA was direct or indirect (that is, requiring $\mathrm{NF}-\kappa \mathrm{B}-$ dependent transcription). MyoD mRNA decay rates, de- termined in experiments in which transcription was blocked with actinomycin $\mathrm{D}$, were similar whether $\mathrm{C} 2 \mathrm{C} 12$ myoctyes were treated or not treated with TNF (16), which suggests that regulation of MyoD is dependent on an NF-кB-responsive gene. To test the requirement for the transcription function of p65 in the regulation of $M y o D$, we performed reporter assays in mouse 10T1/2 fibroblasts with deletion mutants lacking regions of the p65 transactivation domain. Deletion of the entire transactivation domain of p65 [Fp65(313)] restored MyoD function (Fig. 3C). These data are consistent with those in Fig. 2A, where the p50 subunit of NF- $\mathrm{BB}$, which lacks a transactivation domain, was also unable to inhibit MyoD expression. Taken together, these data indicate that $M y o D$ mRNA is inhibited at the posttranscriptional level in a manner requiring $\mathrm{NF}-\kappa \mathrm{B}-$ dependent transcription.

TNF is an important mediator in the skeletal muscle degeneration associated with cancer-induced cachexia (10-12). Direct inhibition of NF- $\mathrm{KB}$ also blocks cachexia in an animal model (17), suggesting a link between $\mathrm{NF}-\kappa \mathrm{B}$ and TNF in this disease. Although delivery of TNF is sufficient to induce weight loss in animals, in vitro studies showing that skeletal muscle explants incubated with TNF do not undergo degeneration (18) support the notion that at least one other factor is required to induce muscle wasting. Consistent with these findings, we found that in contrast to the effect of TNF on differentiating myoblasts, differentiated myotubes were completely refractory to this cytokine with respect to MyoD and MHC expression (Fig. 4A). Because other inflammatory cytokines
Fig. 3. Identification of the MyoD sequences regulated by p65-dependent transcription. (A) p65 inhibits $M y o D$ in a region encompassing nucleotides 539 through 914. The MyoD cDNA was removed from plasmid pEMC11s, containing a long terminal repeat element, and ligated into PCDNA3 (Invitrogen), generating the plasmid $\mathrm{PCMV}$ MyoD. $5^{\prime}$ and $3^{\prime}$ deletions were made in MyoD sequences (16). Numerical values present in the names of each plasmid denote the location of the deletions generated with respect to the $5^{\prime}$ and $3^{\prime}$ ends of the MyoD CDNA. Wild-type MyoD (lanes 1 and 2) or deletion plasmids (lanes 3 through 10), each at $0.5 \mu \mathrm{g}$, were individually transfected in 10T1/2 fibroblasts with or without a p65 expression plasmid $(1.0 \mu \mathrm{g})$. RNA was extracted 24 hours after transfection, and MyoD was probed. Numerical values listed with arrows indicate the predicted sizes of exogenously expressed MyoD mRNA products. (B) p65 inhibits GAPDH expression containing MyoD sequences. Nucleotides 313 through 914 from MyoD were ligated into PCMVGAPDH, generating the plasmid PCMVGAPDH-MyoD. Transfections and RNA analysis were repeated as described in (A). The arrow indicates endogenous GAPDH expression in 10T1/2 fibroblasts. (C) MyoD
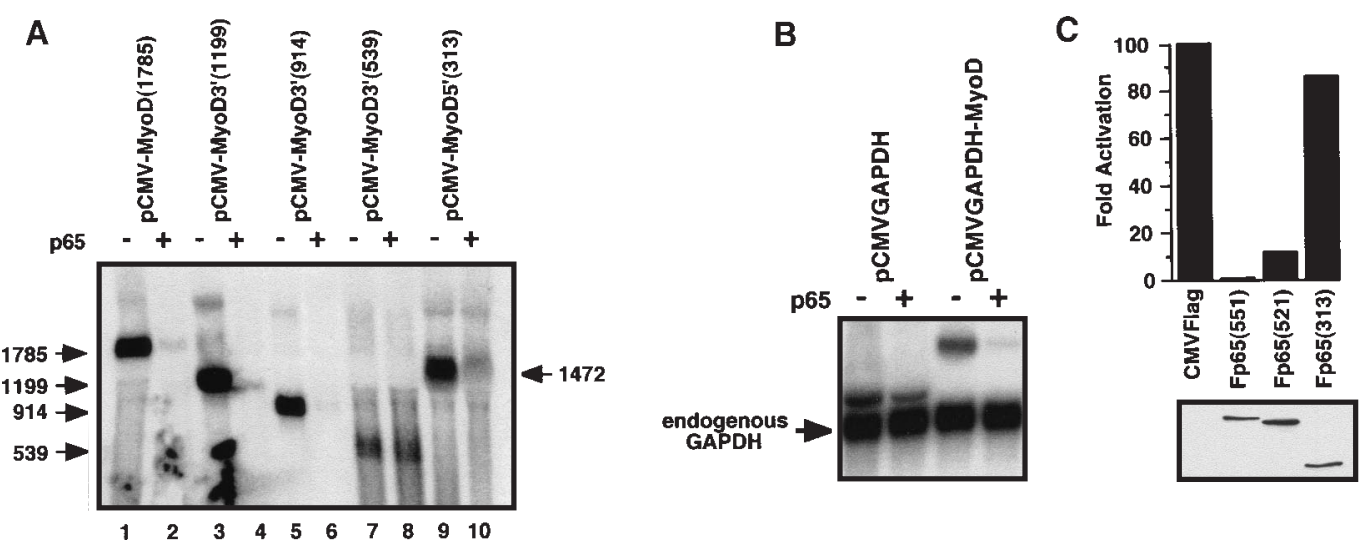

regulation requires p65-dependent transcription. The upper portion shows transfections performed in 10T1/2 fibroblasts with a MyoDresponsive reporter (4RTK-Luc, containing four E-box sites fused to a minimal thymidine kinase promoter), the PCMV-MyoD expression plasmid, and epitope-tagged p65 expression plasmids containing either wild-type [Fp65(551)] or $\mathrm{COOH}$-terminal deletions in the transactivation domain. Cell extracts were prepared with M-Per Extraction Buffer (Pierce) and tested in luciferase assays. The lower portion shows cell extracts from transfections that were probed with an antibody to FLAG (M2, Sigma) to verify the presence of equal p65 expression. 
such as interleukin-1 $\beta$ (IL-1 $\beta$ ), IL-6, and IFN- $\gamma$ also induce cachexia in animal models (19), we investigated the possibility that one of these cytokines in combination with TNF could affect skeletal muscle cellular function. Treatments with IL- $1 \beta$ or IL- 6 alone or in combination with TNF had no effect on skeletal muscle-specific gene expression (Fig. 4A) (15). In addition, IFN- $\gamma$ treatment alone had no effect (15). However, differentiated myotubes treated with TNF plus IFN- $\gamma$ exhibited significant reductions in both MyoD and MHC protein expression (Fig. 4A). Consistent with these results, immunostaining analysis displayed dramatic diminishment of $\mathrm{MHC}$ after treatment with TNF plus IFN- $\gamma$ (Fig. 4C) (16), which correlated with the inability of myofibers to maintain their contractile activities in culture (15). Apoptosis was observed in cultures treated with TNF plus IFN- $\gamma$, but only in those myocytes unable to complete their differentiation program (16), indicating that the loss of muscle cell structure was not a result of cell death. Thus, IFN- $\gamma$ and
TNF together regulate myotube degeneration. When $\mathrm{C} 2 \mathrm{C} 12$ myotubes devoid of NF$\kappa \mathrm{B}$ activity were treated with TNF plus IFN- $\gamma$, they maintained both MyoD and MHC expression (Fig. 4, B and C), indicating that repression of $M y o D$ and impairment of skeletal muscle function are NF$\kappa \mathrm{B}-$ dependent processes.

To test whether TNF and IFN- $\gamma$ regulate $M y o D$ expression in vivo, we injected cytokines directly into mouse skeletal muscle. MyoD mRNA levels were substantially reduced after injection of the cytokines (Fig. 4D). A mixture of tumor-forming Chinese hamster ovary (CHO) cells expressing TNF (12) and IFN- $\gamma(20)$ was also injected into the skeletal muscle of nude mice to more accurately mimic a cachetic state. Again, these cytokines caused a pronounced decline in $M y o D$ mRNA levels but had no effect on GAPDH mRNA (Fig. 4D). These results suggest that $M y o D$ is an in vivo target of cytokine signaling.

In adult skeletal muscle, MyoD is expressed at relatively low levels $(21,22)$.
Fig. 4. IFN- $\gamma$ is required in addition to TNF to regulate skeletal muscle dysfunction. (A) MyoD and $\mathrm{MHC}$ were reduced in myotubes by treatment with TNF plus IFN- $\gamma$. C2C12 myocytes were differentiated for a 3-day period. DM was replaced with either no additive (lane 1); TNF at concentrations of 20 or $100 \mathrm{ng} / \mathrm{ml}$ (lanes 2 and 3 , respectively); TNF (20 ng/ml) plus IL-1 $\beta$ (20 ng/ml; Promega) (lane 4); TNF (20 ng/ml) plus IL-6 (20 ng/ml; Promega) (lane 5); or TNF (20 $\mathrm{ng} / \mathrm{ml}$ ) plus IFN- $\gamma$ (100 U/ml; Life Technologies) (lane 6).

Treatments were repeated at 6,12 , and 24 hours. At 48 hours, whole-cell lysates were prepared and probed for MyoD, MHC, and $\alpha$-tubulin. (B through D) Regulation of MyoD and myosin by TNF and IFN- $\gamma$ is NF- $\kappa B$-dependent. Vector control or I $\mathrm{I}_{\mathrm{B}} \alpha \mathrm{SR}$ expressing myoblasts were differentiated as described in (A) and then treated with TNF and/or IFN- $\gamma$ for 48 hours. Lysates were prepared and probed for MyoD and MHC (B), or cells were immunostained (24) for MHC (C). Scale bar, $15 \mu \mathrm{m}$. (D) MyoD was down-regulated by cytokines in vivo. Upper panels: BALB/C and $\mathrm{B} 6 / 129$ mice were given saline alone (vehicle) or a combination of $2 \mu \mathrm{g}$ of TNF and $5000 \mathrm{U}$ of IFN- $\gamma$ in a volume of 0.1 $\mathrm{ml}$ in the right gastrocnemius muscle. Injections were repeated every 4 hours. At 12 hours, gastrocnemius muscles were dissected and quick-frozen in liquid nitrogen. At a later time, RNA was prepared with the use of TRIZOL reagent (Life Technologies), and Northern analyses were performed to detect MyoD and GAPDH expression. Lower panels: $1 \times 10^{7}$ vector control $\mathrm{CHO}$ cells or a mixture of $\mathrm{CHO}$ cells expressing human TNF (12) and mouse IFN- $\gamma$, clones 1 and 15 (20), were injected into right gastrocnemius muscles of nude mice. At day 12 after injection, gastrocnemius muscles were isolated, RNA was prepared, and Northern analysis was performed, probing for MyoD and GAPDH.
However, in response to injury, MyoD expression is induced from satellite cells, and genetic evidence clearly demonstrates that MyoD is required for these cells to proliferate and to reinitiate SMD necessary for the repair process $(4,21)$. Our data demonstrate that TNF, along with a second proinflammatory cytokine, IFN- $\gamma$, functions through NF- $\kappa \mathrm{B}$ to suppress MyoD synthesis by repressing the accumulation of $M y o D$ mRNA. With respect to an injury model such as cachexia, we propose that the cytokines TNF and IFN- $\gamma$ are likely to affect skeletal muscle regulation at two phases: first, when TNF alone would be sufficient to suppress MyoD expression, thereby inhibiting the formation of new myofibers; and second, when combined signaling between TNF and IFN- $\gamma$ would be required to inhibit MyoD and cause the degeneration of newly formed myotubes. The combined cytokine effects, working through NF- $\mathrm{BB}$, would lead to the inability to repair damaged skeletal muscle, thereby contributing to the overall wasting process associated with cachexia. Thus, direct inhibition of NF- $\mathrm{KB}$ may prove beneficial in reducing the muscle wasting associated with cachexia in cancer patients as well as with other disorders.

\section{References and Notes}

1. B. L. Black and E. N. Olson, Annu. Rev. Cell Dev. Biol. 14, 167 (1998)

2. A. B. Lassar, S. X. Skapek, B. Novitch, Curr. Opin. Cell Biol. 6, 788 (1994).

3. M. A. Rudnicki, T. Braun, S. Hinuma, R. Jaenisch, Cell 71, 383 (1992)

4. L. A. Megeney, B. Kablar, K. Garrett, J. E. Anderson, M. A. Rudnicki, Genes Dev. 10, 1173 (1996).

5. S. Baghdiguian et al., Nature Med. 5, 503 (1999).

6. D. C. Guttridge, C. Albanese, J. Y. Reuther, R. G. Pestell, A. S. Baldwin, Mol. Cell. Biol. 19, 5785 (1999).

7. S. Ghosh, M. J. May, E. B. Kopp, Annu. Rev. Immunol. 16, 225 (1998).

8. A. S. Baldwin, Annu. Rev. Immunol. 14, 649 (1996).

9. D. Thanos and T. Maniatis, Cell 80, 529 (1995).

10. K. J. Tracey and A. Cerami, Annu. Rev. Med. 45, 491 (1994).

11. J. M. Argiles and F. J. Lopez-Soriano, Med. Res. Rev. 19, 223 (1999).

12. A. Oliff et al., Cell 50, 555 (1987).

13. J. van Eys, Annu. Rev. Nutr. 5, 435 (1985).

14. K. Szalay, Z. Razga, E. Duda, Eur. J. Cell Biol. 74, 391 (1997).

15. D. C. Guttridge and A. S. Baldwin, unpublished data.

16. Supplemental figures and information are available at www.sciencemag.org/feature/data/1052671.shl.

17. I. Kawamura et al., Gene Ther. 6, 91 (1999).

18. L. L. Moldawer, G. Svaninger, J. Gelin, K. G. Lundholm, Am. J. Physiol. 253, C766 (1987).

19. M. J. Tisdale, J. Natl. Cancer Inst. 89, 1763 (1997).

20. $\mathrm{CHO}$ cells were transfected with an IFN- $\gamma$ expression plasmid along with a gene conferring neomycin resistance. Clones were obtained with neomycin treatment $(500 \mu \mathrm{g} / \mathrm{ml})$ and screened for cytokine expression by sandwich enzyme-linked immunosorbent assay (Endogen, MA) and Northern analysis. Two clones were analyzed.

21. R. N. Cooper et al., J. Cell Sci. 112, 2895 (1999).

22. K. Koishi, M. Zhang, I. S. McLennan, A. J. Harris, Dev. Dyn. 202, 244 (1995).

23. Mouse $10 \mathrm{~T} 1 / 2$ fibroblasts were grown overnight in triplicate in $60-\mathrm{mm}$ culture dishes. Cells were then transfected by means of Superfect (Qiagen) with $160 \mathrm{ng}$ of either empty vector or a MyoD expression plasmid 
( $\mathrm{pEMC11s)}$ alone, or in combination with $300 \mathrm{ng}$ each of input plasmids. After 24 hours, cells were switched to differentiation medium (DM) for 48 hours and wholecell extracts were subsequently prepared.

24. For immunofluorescence microscopy, cells were fixed directly in the culture wells for $30 \mathrm{~min}$ at room temperature with $4 \%$ paraformaldehyde diluted in phosphate-buffered saline (PBS). The cells were washed three times with PBS and then permeabilized for $5 \mathrm{~min}$ with $0.1 \%$ Triton $\mathrm{X}-100$ and sodium citrate and blocked washes with PBS, cells were incubated for $60 \mathrm{~min}$ at room temperature with an antibody to anti-skeletal MHC (1:250, Sigma) diluted in 3\% bovine serum albumin and PBS. After washes, cells were incubated for 30 min with a rhodamine-conjugated antibody to mouse immunoglobulin G (1:100 Oregon Red, Molecular Probes) diluted in $10 \%$ goat serum and PBS. Cells were kept in PBS to be photographed on a Olympus $1 \times 70$ fluorescence microscope.
25. We thank members of the late H. Weintraub's laboratory as well as S. Konieczny, D. Wang, and B. Martin for plasmids; D. Defeo-Jones for CHO-expressing TNF cells; and J. C. Cusack and R. Liu for animal assistance. Supported by NIH grants Al35098 and CA72771 (to A.S.B) and K01 CA78595 (to M.W.M) and by American Cancer Society postdoctoral fellowship grant 9903801 (to D.C.G).

30 May 2000; accepted 10 July 2000

\title{
Increase of Maximum Life-Span in Sweden, 1861-1999
}

\author{
J. R. Wilmoth, ${ }^{1 *}$ L. J. Deegan, ${ }^{1}$ H. Lundström, ${ }^{2}$ S. Horiuchi ${ }^{3}$
}

\begin{abstract}
A fundamental question in aging research is whether humans and other species possess an immutable life-span limit. We examined the maximum age at death in Sweden, which rose from about 101 years during the 1860 s to about 108 years during the $1990 \mathrm{~s}$. The pace of increase was 0.44 years per decade before 1969 but accelerated to 1.11 years per decade after that date. More than 70 percent of the rise in the maximum age at death from 1861 to 1999 is attributable to reductions in death rates above age 70 . The rest are due to increased numbers of survivors to old age (both larger birth cohorts and increased survivorship from infancy to age 70 ). The more rapid rise in the maximum age since 1969 is due to the faster pace of old-age mortality decline during recent decades.
\end{abstract}

The world record of human life-span seems to be moving upward over time, as suggested by the death in 1997 of Jeanne Calment at the documented age of 122.45 years (1). However, such events are a poor measure of the trend in achieved human life-span, which can be studied more effectively using data for well-defined populations. National demographic statistics suggest that the maximum age at death has been rising steadily in industrialized countries for more than 100 years (2).

Two important questions arise from this observation. First, has this upward trend been steady over time, or has it changed pace in recent years? Perhaps the increase has accelerated due to an intensification of efforts to promote the health of the elderly and to prevent or even cure ailments such as coronary heart disease, stroke, and cancer. Or perhaps the trend has decelerated because maximum ages now observed for humans are approaching a (hypothetical) biological limit. Second, what accounts for the increase in the maximum age at death? There are two competing explanations. One is that it is due merely to the larger size of contemporary populations, which increases the probability that at least

'Department of Demography, University of California, Berkeley, CA 94720-2120, USA. 'Statistics Sweden, Box 24300, S-104 51, Stockholm, Sweden. 'Laboratory of Populations, Rockefeller University, 1230 York Avenue, New York, NY 10021-6399, USA.

*To whom correspondence should be addressed. Email: jrw@demog.berkeley.edu one individual will survive to an extreme old age. Another possibility is that the increase reflects improvements in an individual's probability of survival, especially at older ages.

We have investigated these questions using Swedish national demographic data from 1861 to 1999 , which are the longest available series of reliable information on the upper limits of achieved human life-span. Recorded maximum ages at death for men and women in Sweden centered around 101 years during the 1860 s and around 108 years during the 1990s (Fig. 1). A statistical analysis indicates that the increase in the maximum age at death accelerated markedly around 1969 , rising at a rate of 0.44 years per decade from 1861 to 1969 and 1.11 years per decade from 1969 to 1999. In both time periods, the maximum age at death was on average about 1.7 years lower for men than for women, although time trends for both sexes are similar (3).

Apparent trends in the maximum age at death can be distorted by changes in data quality. Inaccurately reported ages of very old persons and decedents have been common in official statistics for many countries $(2,4)$. An improvement in data quality (i.e., fewer exaggerated reports of extreme old age) may lead to a decrease over time in the maximum reported age at death. Thus, a typical pattern is that the maximum age may decline for several years as data quality improves and then begin to increase. Only the latter increase, not the earlier decrease, reflects a true trend. Mortality data since 1861 in Sweden show none of the typical signs of age misreporting that are common elsewhere (2). Mortality histories for the countries of Western Europe and North America are largely similar to the Swedish experience, but no other country's data offer the possibility for reliable trend analysis in extreme old age over such a long period. For this reason, only Swedish data are used here, although the results should apply broadly to the populations of other highly industrialized countries.

We analyzed the effect of changes in demographic factors (birth counts and age-specific mortality rates) on the trend of the maximum age at death with the use of a model that treats the observed maximum age as a random variable with a theoretical probability distribution. This distribution is determined both by the underlying distribution of ages at death for individuals and by the initial size of a birth cohort (5). For this analysis, data on the maximum age at death were reorganized by year of birth (cohort) rather than by year of death (period) (Fig. 2). These two trends are similar and contain many of the same data points. However, some points appear in one series but not the other, because a death that qualifies as the oldest for a birth cohort may or may not be the oldest in the year of death, and vice versa.

Our analysis of the cohort trend in the maximum age at death relies on a reconstruction of Swedish mortality by year of birth back to 1751 . The life tables and the methodology used to produce them are available on our Web site (6). Although there may be some question about the quality of period life tables from late 18th-century Sweden (especially at older ages), life tables for cohorts born in this era are more reliable because the quality of the statistical system improved over their lifetime. By the time such cohorts attained age 100 or 110 , where problems of data quality are most severe, the Swedish statistical system was extremely accurate (7).

In order to determine probability distributions of the maximum age at death, a sequence of age-specific death rates for each cohort is needed. Death rates below age 80 were computed directly from national mortality statistics, reorganized in a cohort format (8). Death rates above age 80 were estimated by fitting a logistic function to observed death rates and then extrapolating this function to very high ages (9). It was neces- 
NF-kB-Induced Loss of MyoD Messenger RNA: Possible Role in Muscle Decay and Cachexia

Denis C. Guttridge, Marty W. Mayo, Lee V. Madrid, Cun-Yu Wang and Albert S. Baldwin Jr.

Science 289 (5488), 2363-2366.

DOI: $10.1126 /$ science.289.5488.2363

ARTICLE TOOLS

RELATED
CONTENT

REFERENCES

PERMISSIONS http://science.sciencemag.org/content/289/5488/2363

http://science.sciencemag.org/content/sci/289/5488/2293.full

This article cites 19 articles, 3 of which you can access for free http://science.sciencemag.org/content/289/5488/2363\#BIBL

http://www.sciencemag.org/help/reprints-and-permissions

Use of this article is subject to the Terms of Service

Science (print ISSN 0036-8075; online ISSN 1095-9203) is published by the American Association for the Advancement of Science, 1200 New York Avenue NW, Washington, DC 20005. 2017 @ The Authors, some rights reserved; exclusive licensee American Association for the Advancement of Science. No claim to original U.S. Government Works. The title Science is a registered trademark of AAAS. 